\title{
Neonatal Abstinence Syndrome
}

\author{
Kirtisudha Mishra K ${ }^{1}$, Chopra $N^{2}$, Dudeja $A^{3}$, Datta $V^{4}$, Saili $A^{5}$, Dutta AK
}

${ }^{1-6}$ Department of Paediatrics

Lady Hardinge Medical College and associated Kalawati Saran Children's

Hospital, New Delhi, India

\section{Corresponding Author}

Dr Kirtisudha Mishra

Flat 9, Plot 6, Manav Vihar, Sector 15, Rohini

New Delhi, India

Email. kirtisen@gmail.com

\begin{abstract}
Intrauterine exposure to drugs by mothers is not an uncommon finding ine our society. Due to the mother's suppression of a medical history, the diagnosis of neonatal abstinence syndrome is often missed. We report a case of a term, female, newborn, who presented with the following features; e restlessness, inconsolable crying, along with sweating, vigorous sucking; andadiarrhoea. No conclusion was derived from routine investigations. Eventually, with a high degree of suspicion regarding maternal drug addiction, her history was reviewed and it was discovered that the mother was a heroin addict. The baby was diagnosed as a case of Neonatal Abstinence Syndrome. The neonate was successfully managed thereafter and discharged.
\end{abstract}

\section{Key Words}

neonatal abstinence syndrome, neonate,

\section{INTRODUCTION}

Neonatal Abstinence Syndrome(NAS) is a growing problemastheprevalenceofdrugabuseamongwomen ofthechild-bearingagegroupisincreasingovertheyears $\therefore$.'Asadiagnosis, itislargelyunder-reportedfrom South East Asia. This is due to the lack of disclosure of drug abuse, andalsothesymptomsaremostlynon-specific, hencethediagnosisisoftenmissed.Averyhighindex of suspicionandfocusedhistorytakingisrequiredtoidentify suchcases.WearereportingacaseofNAS duetoHeroin Withdrawal, whichwastimelydiagnosedandsuccessfully managed.

\section{CASE REPORT}

Afulltermfemalenewbornweighing2.2Kkgwasbornby NormalVaginaldeliveryinaTertiaryCareMedicalCentre, withanApgarscoreof7,8atfirstandfifthminuteofbirth respectively.TheMmotherwasa28yearold, unbooked case, Para3 three with three living issues. Her HIV, VDRL andHepatitisBstatuswastestedafterdeliveryandfoundto benegative.Parentswerebothstreetdwellers.Thebaby's birthweightwas $2.25 \mathrm{~kg}(<10$ thpercentile), length $46 \mathrm{~cm}$ $(<10$ thpercentile), andheadcircumference $33 \mathrm{~cm}(<25$ th percentile.Themotherwasmalnourishedandanaemic.
During initial questioning, no history was available regarding any form of addictio. At two hours of life, the babydevelopedfeverandtachypneaandwasadmittedin thenursery.Onadmission, thepatient'stemperaturewas $38^{\circ} \mathrm{C}$,respiratoryrate $66 / \mathrm{min}$, heartrate $142 / \mathrm{min}$,capillary fillingtimelessthan3threeseconds(notprolonged),blood pressure66/46 (mean 53) $\mathrm{mm}$ of Hg. Subsequently, the babyalso developed an abnormalrbehaviour pattern, consisting of excessivehigh-pitchedcrying, irritability, inconsolability,sweatingandadiarrhoea.Babyhadvigorous suckingandanexaggeratedMoro'sreflex.Onthebasisof theseclinicalfeatures,adifferentialdiagnosisofsepsiswith meningitis,intracranialehaemorrhage, ahypocalcaemia,ah ypoglycaemia and thyrotoxicosis was entertained.

Investigations revealed a negative septic screen, CSF analysis,bloodsugarandserumelectrolyteswereallwithin normallimits.ChestX-rayandultrasoundskullshowed noabnormalityandthyroidfunctiontestwasalsonormal. This led to a diagnostic dilemma.

Thehistorywasreviewedagainanditwasdiscoveredthat themotherwasa)heroinaddictforthepast5fiveyears.She hadbeentakingthedrugbyinhalation,duringconception, and throughout pregnancy. Taking into account the history, clinical presentation and a normal laboratory profile,thepatientwasdiagnosedasacaseofeNASdueto 
heroin withdrawal.

The patient was managed symptomatically and the Neonatal Abstinence Scoring System (NASS) ${ }^{2}$ was meticulously followed. Within 24 hours of life of the baby, three consecutive NASS scores showed values greaterthan8eightandphenobarbitonewasstartedt3mg/ $\mathrm{Kg} /$ day (Fig.1). The maximum NASS score reached 12onday3 threeoflifeand the doseofphenobarbitone was increased to $8 \mathrm{mg} / \mathrm{Kg} /$ day, after which, symptoms graduallydecreasedand phenobarbitonewasgradually tapered offand discontinued byday 10 oflife.TheBbaby was discharged afterg the mother was referred ton rehabilitation and counselling centrr.

\section{DISCUSSION}

The incidence of drug-exposed newborns has been reported to vary from $3 \%$ to $50 \%$, depending on the specificpatientpopulation, withurbanscentrestending toreporthigherrates. ${ }^{3}$ Amongedrug-exposednewborns, the incidence ofeNAS ranges from $60-87 \%, 4,5,6$ though a study from the U.K. reported an incidence of 21 . The majordrugs ofabusecan beclassified into fourgroups. Theyare-(I)Opiateesuchasmorphine, methadone, and heroin; (II) CNS stimulantse such as amphetamines an cocaine;(III)CNS depressantslikealcohol,barbiturates, benzodiazepines; (IV) and lastly Hallucinogens such as LSD. ${ }^{3}$ The features of narcotic withdrawal have been summarizedinTable1. ${ }^{8}$ Theonsetofwithdrawalsymptoms varsfrom6twotosixhoursfwithdiazepam,to4oneto 144 hours withf heroin. ${ }^{9}$ Similarly, the duration ofeNAS) is variablefordifferentdrugs. Neonateswhodonotexhibit symptomsofdrugwithdrawal withinthefirstthreedaysof lifeareunlikelytopresentwithNASrequiring treatment. ${ }^{6}$ Diagnosing NAS can bs made by screening tests in the meconiumorurineofthenewborn.Urinescreeninghas ahighfalsenegativeratebecauseonlyresultsforinfants withrecentexposurewilletestaspositive.Meconiumdrug testing,althoughnotconclusiveifresultsarenegative, is moreaccuratethanurinesampleoinidentifyinginfants ofdrug-usingmother.Meconiumanalysiswasfoundtoe have $96 \%$ sensitiviteand $77 \%$ specificity. ${ }^{10}$ Thedifferential diagnosis includes central nervous system infections, metabolicdisorderssuchasahypoglycaemia,ahypocalcaem ia, intracranialehaemorrhageandthyrotoxicosis. Theaim ofmanaging babieswhosufferfromNASistohaveanonirritablebabywithoutvomitingoradiarrhoea, whofeeds well,sleepswellbetweenfeedsandisnotheavilysedated. Currentlyitisstated thattheadministration ofnaloxone toan infant ofanarcotic-addicted mothermayresultin abruptdrugwithdrawalandseizures. ${ }^{2}$ Inthepresentcase reported, themotheroftheneonatewasaheroinaddict.
Heroin is an opioid and naloxone is a pure competitive opioidantagonist.Ssoasaresult,treatmentwithnaloxone could precipitateimmediatewithdrawalsymptomsand seizures. Managementofsuchsymptomsincludsetight swaddlin;rocking, avoidanceofexcessivelightandsound; fluid and electrolyte maintenance, monitoring of the newborn'seclinicalconditionbyapplicationofthe(NAS) ; and lastly management of the social aspects. The NASSisbasedonvitalparameters,sleeping patterns, the newborn'scentralnervoussystem, andtheautonomicand gastrointestinaldisturbancesofthynewborn.TheMmost commonlyuseddrugsfortreatment ofNASisneonatal morphinesolution $(0.4 \mathrm{mg} / \mathrm{ml})$, adoseofwhichistitrated according to the newborn's NASS scores. ${ }^{9}$ Other drugs areneonatalopiumsolution,paregoric, phenobarbitone, chlorpromazine,anddiazepam. However,thereisstillittle evidenceregardingtheefficacyofthedifferenttherapeutic regimes.lthasbeenfoundthatmorphinetreatednewborns requireasignificantlyshorterdurationoftreatmentversus those treated with phenobarbitone. ${ }^{5}$

Drugsofabusethatarecontraindicatedforusebymothers (who are already taking drugs) during breast-feeding include amphetamines, cocaine, heroin, marijuana, nicotine and phencyclidine. Drugs that should be used cautiously if a woman is breast-feeding include phenobarbital and benzodiazepines. Methadone is compatiblewithbreast-feedingatadoseoflessthan20mg every24hours.Ithasalsobeen proventhatdrugexposure toeanursinginfantcanbeminimisedifthemothertakes medicationjustaftercompletingnursingorjustbeforean anticipated lengthy sleep period. ${ }^{11}$

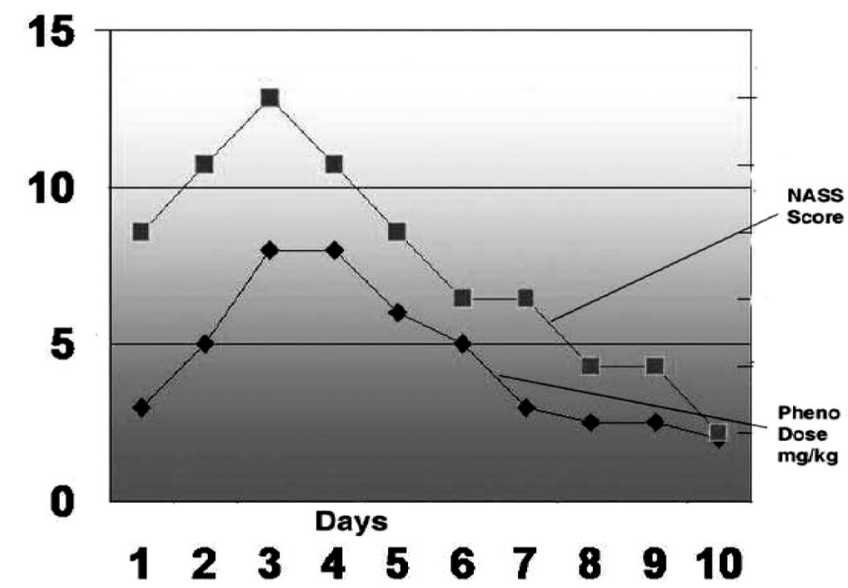

Figure 1. Agraphicalrepresentation of therelationship between dose of phenobarbitone and NASS scores 
There is limited information regarding the prognosis and long-term consequences of perinataladdiction. It is also difficult toattributeneurologicaldeficitstodrug effects or to environmental conditions due to overlap .Severalstudieshaveidentifiedincreasedneurological abnormalities such as articulation disorders, weak visual, motor and perceptual skills, and disruptive and aggressivelbehavioural problems. ${ }^{11}$

\section{REFERENCES}

1. Mirjana V, Damir R, Zoran V, Vesna C, Marija B, Ivo B.Maternal and Neonatal Effects of Substance Abuse duringPregnancy:OurTen-yearExperience.YonseiMedJ 2008;49:705-13.

2. FinneganLP,KaltenbachK.Neonatalabstinencesyndrome. In: Hoekelman RA, Friedman SB, Nelson N, Seidel HM, editors. Primary pediatric care. 2nd ed., St Louis:CV Mosby;1992.p.1367-78.

3. American Academy of Pediatrics, Committee on Drugs. Neonataldrugwithdrawal.Pediatrics1998;101:1079-1088.

4. Blaser A, Pulzer F, Knupfer M et al. Drug Withdrawal in Newborns-ClinicalDataof49InfantswithIntrauterineDrug Exposure: What should be Done? KlinPadiatr 2008;220: 308-315.

5. EbnerN,RohrmeisterK,WinklbaurBetal.Managementof neonatalabstinencesyndromeinneonatesborntoopioid maintainedwomen.DrugAlcoholDepend2007;87:131-8.

6. SeraneVT,KurianO.Neonatalabstinencesyndrome.Indian J Pediatr 2008;75:911-4 .

\section{CONCLUSION}

These children are at risk of child abuse and neglect. Identificationofthesehigh-riskinfantsandappropriate intervention is critical. Organizations, which can specifically look into the needs of these compromised mothersandtheirchildren, areindesperateneedinNepal..
7. Mayet S, Groshkova T, Morgan L, MacCormack T, Strang J.Drugsandpregnancy-outcomesofwomenengagedwith specialistperinataloutreachaddictionsservice.DrugAlcohol Rev 2008;27:497-503.

8. RiversR.Thebabyofasubstance-abusingmother.InJanet MRennie,ed.Roberton'sTextbookofNeonatology.4thedi. London:Elsevier Limited;2005.p.433-42.

9. Schechner S. Drug Abuse and withdrawal. In:Cloherty JP, Eichenwald EC, Stark ER. editor. Manual of neonatal Care 5th edi. Philadelphia:Lippincot Williams and Wilkins,:2004.p.223-36.

10. Ostrea EM Jr, Martier 5, Welch R, Brady M. Sensitivity ofmeconium drugscreen in detecting intrauterinedrug exposure of infants. Pediatr Res 1990;27:219A

11. GHoegerman, CAWilson, EThurmond, and SH Schnoll. Drug-exposed neonates. West J Med. 1990;152:559-64. 